\title{
The Quantification of Glycosaminoglycans: A Comparison of HPLC, Carbazole, and Alcian Blue Methods
}

\author{
Sarah B. Frazier ${ }^{1}$, Kevin A. Roodhouse ${ }^{1}$, Dennis E. Hourcade ${ }^{2}$ and Lijuan Zhang*,1 \\ ${ }^{I}$ Department of Pathology and Immunology, ${ }^{2}$ Division of Rheumatology and Department of Medicine, Washington \\ University School of Medicine, St. Louis, MO 63110, USA
}

\begin{abstract}
Glycosaminoglycans (GAGs) are linear polysaccharides that are found in the extracellular matrix and biological fluids of animals where they interact with hundreds of proteins and perform a variety of critical roles. There are five classes of animal GAGs: heparan sulfate (HS), chondroitin sulfate (CS), dermatan sulfate (DS), keratan sulfate (KS), and hyaluronan (HA). Many biological functions can be monitored directly by their impact on GAG quantity. Thus, simple, sensitive, and robust GAG quantification methods are needed for the development of biomarkers. We have systematically compared three available GAG quantification assays including an HPLC-based assay, a simplified Alcian Blue assay, and a miniaturized carbazole assay. The carbazole and Alcian Blue assays were reproducible and simple to perform in general lab settings, but had important limitations: The carbazole assay could not detect KS and it overestimated GAGs that were contaminated with salts or dissolved in PBS. The Alcian Blue assay detected only those GAGs that were sulfated. In contrast, while the HPLC method was time-consuming, it was a robust and sensitive assay that not only detected all GAGs but also quantified glucosamine-GAGs and galactosamine-GAGs simultaneously. The HPLC assay was not affected by salt or level of GAG sulfation and it yielded reproducible values for all types of GAGs tested. These results suggest that an automated HPLC assay would be generally useful for the routine measurement of a panel of GAG-based biomarkers while the carbazole assay and the Alcian Blue assays could prove valuable for more specific purposes.
\end{abstract}

\section{INTRODUCTION}

Glycosaminoglycans (GAGs) are linear polysaccharides comprised of repeating hexosamine-containing disaccharides that are sulfated to varying degrees. Most animal cells synthesize multiple types of GAGs including heparan sulfate (HS), chondroitin sulfate (CS), dermatan sulfate (DS), keratan sulfate (KS), and hyaluronan (HA). HS, CS, and DS are assembled on proteoglycan core proteins in the Golgi through linkage tetrasaccharides (GlcA-Gal-Gal-Xyl-Ser). $\mathrm{KS}$ is assembled on specific core proteins through $\mathrm{N}$-linked (KSI) or O-linked (KSII) oligosaccharides. HA is unique in that it is unsulfated and is directly secreted without a core protein into the extracellular space.

HA and HS consist of repeating disaccharide units of GlcNAc and GlcA while KS is composed of alternating Nacetyl glucosamine (GlcNAc) and galactose. CSs consist of alternating GalNAc and GlcA/IdoA. They are classified as $\mathrm{CS}$ types $\mathrm{A}, \mathrm{B}, \mathrm{C}, \mathrm{D}$, and $\mathrm{E}$ according to the predominant disaccharide form (Table 1). CSs isolated from animal tissues usually feature one major type of repeating disaccharide, but always contain minor species of other disaccharides. DS, also known as CS-B, is distinguished from other CS varieties by its high content of IdoA residues and its unique anticoagulant properties [1].

The most studied GAG, heparin, is a heavily sulfated heparan sulfate made by mast cells. Heparin is used clini-

*Address correspondence to this author at the Department of Pathology and Immunology, Washington University School of Medicine, St. Louis, MO 63110, USA; Tel: 314-362-8850; Fax: 314-362-3016;

E-mail: ljzhang@wustl.edu cally as an anticoagulant drug but also possesses anti-sepsis [2], anti-spontaneous abortion [3], anti-selectin mediated inflammation, anti-interstitial cystitis, and anti-tumor metastasis properties [4-8]. Commercial heparin is expressed as units/mg of anticoagulant activity because both its purity and quality are difficult to control. CS and glucosamine are also of clinical interest, ranking third among all top-selling nutriceuticals in the United States as a supplement for treating osteoarthritis [9].

Many biological functions can be monitored directly by their impact on GAGs since hundreds of proteins interact with GAGs [10]. GAGs are directly involved in many signaling pathways [11]. In addition, GAG biosynthesis is regulated by a variety of chemokines, cytokines, and growth factors $[12,13]$. Thus, simple, sensitive, and robust GAG quantification methods are needed for the development of biomarkers.

GAGs can be quantified and analyzed by NMR [14], MS [15], LC-MS [16], enzymatic post column fluorescence HPLC [17], electrophoretic methods [18], and by more general methods for monosaccharide composition analysis such as high-performance anion-exchange chromatography (HPAEC) with pulsed amperometric detection (PAD) [19]. However, a simple, direct, sensitive, and robust quantification method is needed for routine GAG quantification.

Three direct GAG quantification methods, including HPLC [20], carbazole [21,22], and Alcian Blue [23] assays have been established. However, a systematic comparison of these three assays in terms of sensitivity, reliability, and limitations has not been reported. We have systematically quantified CS-A, CS-B, CS-C, CS-D, CS-E, completely 
Table 1. Disaccharide Content of Various Chondroitin Sulfates

\begin{tabular}{|c|c|c|}
\hline Major disaccharides & \multicolumn{2}{|c|}{ Other disaccharides found } \\
\hline \hline CS-A: GlcA-GalNAc4S & GlcA-GalNAc & IdoA2s-GalNAc \\
\hline CS-B: IdoA2S-GalNAc4S & IdoA-GalNAc6S & GlcA3S-GalNAc \\
\hline CS-C: GlcA-GalNAc6S & IdoA-GalNAc4S6S & GlcA3S-GalNAc4S6S \\
\hline CS-D: GlcA2S-GalNAc6S & IdoA2S-GalNAc4S6S & GlcA3S-GalNAc6S \\
\hline CS-E: GlcA-GalNAc4S6S & IdoA2S-GalNAc & \\
\hline
\end{tabular}

desulfated re-N-acetylated (CDSNAc)-heparin, N-desulfated re-N-acetylated (NDSNAc)-heparin, and heparin with our previously published HPLC assay [20], a simplified Alcian Blue assay [24], and a miniaturized carbazole assay. We examined the performance of each assay with sulfated and de-sulfated GAGs and in the presence of salt and DNA. The HPLC method detected and quantified all the GAGs we tested and was unaffected by salt or DNA contaminants. In addition, the HPLC method detected cross contaminations of certain glucosamine- and galactosamine-GAGs. The Alcian Blue assay could only detect sulfated GAGs. Contrary to previous reports, we found that the carbazole assay produced false positive signals due to the presence of salt. This information may prove useful in the design of simple GAG-based assays.

\section{RESULTS \\ HPLC Analysis}

GAG preparations are usually a mixture of GAG species due to their biological co-expression and structural similarities. Thus, whether GAGs are isolated, digested, fractionated, or purchased, their purity and concentration must be determined before they are used in biological assays. We previously developed an HPLC-based method that measures all GAGs tested with great precision and accuracy [20]. It can readily detect $10 \mathrm{pmol}$ of GlcN or GalN. Using our standard procedure, we analyzed commercially available CS A, B, C, D, E, and heparin, adding norleucine (NorL) to each GAG sample for normalization purposes. The reduced glucosamine (GlcN-OH), galactosamine (GalN-OH), and NorL profiles of $1 \mu \mathrm{g}$ dry weight-equivalents of different GAGs are shown in Fig. (1). The GlcN-OH peak was derived from the glucosamine-containing GAGs, including $\mathrm{HS}, \mathrm{KS}$, and $\mathrm{HA}$, while the GalN-OH peak was from CS and DS. As expected, the heparin profile had no GalN-OH peak and the CS D and E profiles had no GlcN-OH peak. However, CS-A, CS-B, and $\mathrm{CS}-\mathrm{C}$ were contaminated with glucosamine containing GAGs as evidenced by the GlcN-OH peaks in their profiles (Fig. 1).

We also analyzed additional lots of heparin, CS-A, CS-B, and CS-C from the same source (Sigma) and CS-E from a different manufacturer (Fig. 2). While this Sigma heparin preparation (Porcine mucosa heparin, catalog number H4784, lot 104K1177) had no detectable GalN-OH (Fig. 1), an earlier lot of the same sigma reagent produced a significant GalN-OH peak (Fig. 2). Moreover, the intensity of GlcN-OH peaks in CS-A, CS-B, and CS-C differed between lots. Only one CS-C lot produced a GlcN-OH peak (Fig. 1).
While no GlcN peaks were detected in squid cartilage CS-E from either commercial source, CS-E obtained from Seikagaku (Fig. 1) yielded twice the detectable GAG per unit dry weight than CS-E obtained from Calbiochem (Fig. 2). These observations demonstrated that the purity of commercial GAG preparations is highly variable.

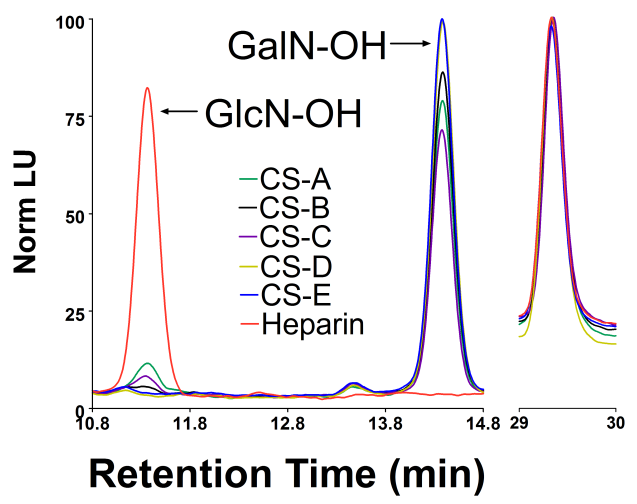

Fig. (1). CS-A, CS-B, CS-C, CS-D, CS-E, and heparin were converted to their respective monosaccharides (GlcN and GalN) via hydrolysis, reduced to GlcN-OH or GalN-OH by sodium borohydride treatment. The OPA derivatives were separated [20]. The elution positions and intensities for $1 \mu \mathrm{g}$ dry weight of $\mathrm{GlcN}-\mathrm{OH}$ and $\mathrm{GalN}-\mathrm{OH}$, and the internal standard norleucine (NorL) are shown. The data is representative of three independent experiments.

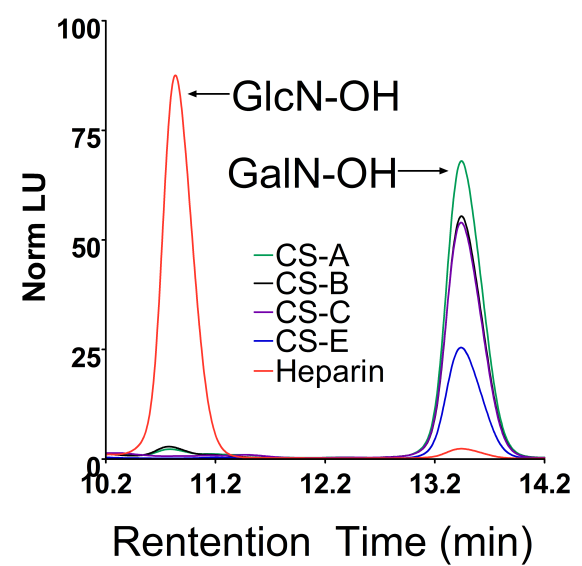

Fig. (2). GlcN-OH and GalN-OH elution profiles of different batches of CS-A, CS-B, CS-C, CS-E, and heparin. The degree of GAG-cross contamination for each GAG differed in a second batch (Fig. 1). In a subsequent experiment, the same degree of contamination was observed for each GAG batch. 
Treatment of HS with $\mathrm{HNO}_{2}$ at $\mathrm{pH} 1.5$ selectively degrades $\mathrm{N}$-sulfated HS and has no effect on CS [25]. We used this reaction to determine whether the putative GlcN peaks derived from CS-B (Fig. 2) were indeed derived from HS contamination. We therefore treated the CS-B preparation with $\mathrm{HNO}_{2}$ at $\mathrm{pH} 1.5$, removed any $\mathrm{HS}$ fragments generated by thorough dialysis, and analyzed the resultant material by HPLC. The profiles of $\mathrm{HNO}_{2}$-treated CS-B and untreated CS-B are shown in Fig. (3A). The $\mathrm{HNO}_{2}$ treatment selectively eliminated the GlcN peak, confirming that the CS-B was indeed contaminated with HS.

We also prepared CS-B oligosaccharides by a partial Ndeacetylation reaction followed by low $\mathrm{pH}$ nitrous treatment of both $\mathrm{HNO}_{2}$-treated CS-B and untreated CS-B. No GlcNcontaining peak was observed in the profiles of any CS-B oligosaccharides prepared from $\mathrm{HNO}_{2}$-treated CS-B (data not shown). However, all the oligosaccharides prepared from the untreated CS-B contained significant amounts of GlcNoligosaccharides (Fig. 3B). The degree of GlcN- oligosaccharide contamination was greatest in the CS-B tetrasaccharides and decreased with increased chain length, but even tetradecasaccharides (CS-B-14) had significantly greater $\mathrm{GlcN}$ content than the original untreated CS-B sample. It appears that HS contamination-levels were greatly amplified in the process of preparing the oligosaccharides. This may be because partially $\mathrm{N}$-deacetylated $\mathrm{CS}-\mathrm{B}$, which has an Nacetylated GlcN content of $100 \%$, is more susceptible to deaminative scission than $\mathrm{HS}$ and heparin, which have an $\mathrm{N}-$ acetylated GlcN content of $60 \%$ and $80 \%$, respectively.
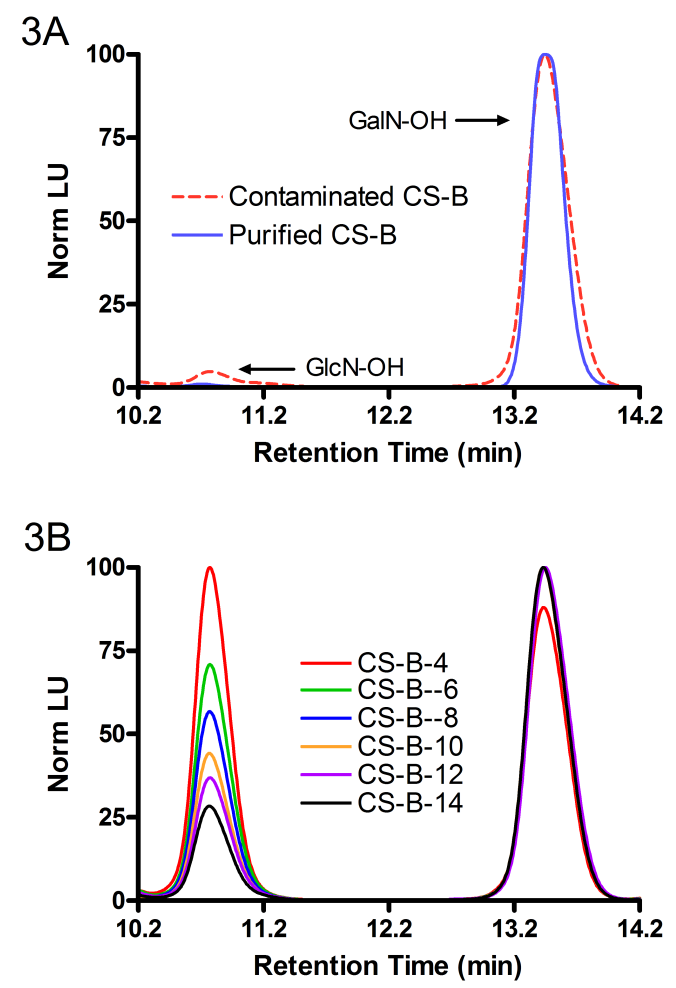

Fig. (3). (A) CS-B was treated with $\mathrm{HNO}_{2}$ at $\mathrm{pH}$ 1.5. After thorough dialysis to remove degraded GAG fragments, the purified CSB was quantified again by the HPLC method. (B) HS oligosaccharides were enriched when contaminated CS-B was used as a starting material for CS-B oligosaccharide preparations.

\section{The Carbazole and Alcian Blue Methods}

The GAGs that were analyzed by the HPLC method (Fig. 1) were then quantified by the carbazole (Fig. 4B) and Alcian Blue (Fig. 4A) assays. Concentration response curves and $r^{2}$ values were determined. The $r^{2}$ values of all the GAGs tested ranged from 0.984 to 0.995 for the Alcian Blue assay and from 0.998 to 0.999 for the carbazole assay, indicating a high degree of reproducibility. However, in both assays, the concentration response curves for the different GAGs were not parallel. This suggests that, in both assays, the quantification reactions were subtly influenced by specific GAG associated properties (structure, impurity, or other factors).
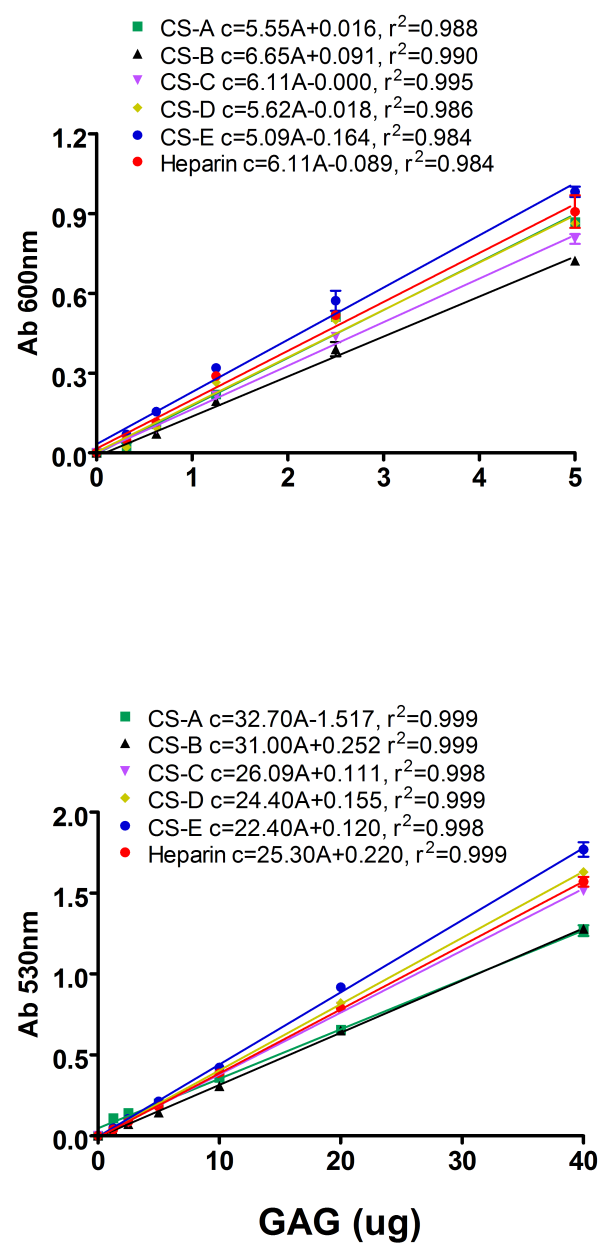

Fig. (4). The CS-A, CS-B, CS-C, CS-D, CS-E, and heparin samples examined by the HPLC method (Fig. 1) were assayed by the Alcian Blue method (panel A) and the carbazole method (panel B). The concentration response curves, equations, and coefficient of correlation $\left(\mathrm{r}^{2}\right)$ for each GAG are representative of over ten independent experiments. Each reaction was performed in duplicate. The variable ' $\mathbf{A}$ ' is the measured absorbance and ' $c$ ' is equal to the quantity of GAGs (in $\mu \mathrm{g}$ ).

\section{Comparison of the Quantification by HPLC \& Carbazole Methods}

The carbazole assay uses GlcA as an external standard. Thus, a calibration curve can be produced for GlcA (Fig. 5) in addition to those generated for the GAG samples (Fig. 
4B). The GlcA curve fitting equation (in this case $\mathrm{c}=26.78 \mathrm{~A}$ +0.4524 with an $\mathrm{r}^{2}$ value of 0.9992 where $\mathrm{A}$ is measured absorbance and $\mathrm{c}$ is the amount of GlcA in nmol) can then be used to calculate the amount of GlcA that corresponds to $1 \mu \mathrm{g}$ for each GAG (Table 2, column 4).

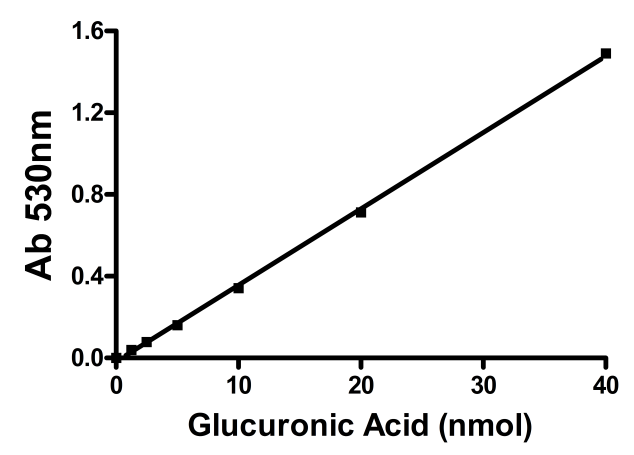

Fig. (5). A 50mM GlcA stock solution was made in water. 0, 1.25, $3.5,5,10$, and $40 \mathrm{nmol}$ dilutions were prepared in duplicate to be used in the generation of an external calibration curve. The curve was used for calculating the GlcA content of the GAG samples. The data shown is representative of more than ten independent experiments.

We then compared the GAG content of each sample based on the GlcA standards (Table 2, column 7), and the GAG content determined by HPLC (Table 2, column 6). As seen in Table 2 , the calculated concentration of nearly all the samples was significantly less than the theoretical GAG stock concentration $(1 \mathrm{mg} / \mathrm{ml})$, which was formulated based on dry weight. This indicates the presence of impurities or water in the GAG samples. Of note, in the case of CS-A, the value obtained with the carbazole assay $(1.27 \mathrm{mg} / \mathrm{ml})$ is greater than its theoretical maximum $(1.00 \mathrm{mg} / \mathrm{ml})$. Moreover, we also noticed that the CS-A concentration response curve ( $c=32.7 \mathrm{~A}-1.517)$ differed significantly from the response curves of the other GAGs (Fig. 4B). CS-A produced a non-linear response, producing relatively high absorbance at low concentrations and relatively low absorbance at high concentrations. These observations led us to conclude that the values yielded by the carbazole assay for the CS-A sample are inaccurate.

\section{In the Carbazole Assay Salts Generated a False Positive}

Comparison of the GAG quantification curves obtained for the carbazole and Alcian Blue assays (Fig. 4) suggests the subtle influence of uncontrolled factors on the quantifica- tion reactions. These discrepancies could simply be due to differences in GAG purity or water content. Nevertheless, we examined the impact of salt on the two assays and observed that $\mathrm{NaCl}$ increased the background absorbance in the carbazole assay in a concentration-dependent manner (Fig. 6A). We calculated that $0.01 \mathrm{mM} \mathrm{NaCl}$ produced an increase in signal intensity equivalent to that generated by $4 \mu \mathrm{g} \mathrm{CS}-\mathrm{A}$ or Heparin. In addition, the GAG and salt signals were additive. In short, the carbazole assay had about 6-fold higher specificity for GAGs than for the $\mathrm{NaCl}$. These results indicated, for example, that the quantity of GAGs dissolved in PBS $(0.15 \mathrm{M} \mathrm{NaCl})$ could be overestimated by up to $60 \mu \mathrm{g} / \mathrm{ml}$ by the carbazole method. In contrast, salt concentrations of up to $1 \mathrm{M} \mathrm{NaCl}$ had minor effects on the Alcian Blue assay (Fig. 6B) and no detectable effects on the HPLC assay (Fig. 7).

\section{DNA Contamination Showed no Effect on the Carbazole or Alcian Blue Assays}

Biological samples, especially GAGs purified from biological sources, may contain DNA. Thus, we examined the impact of DNA contamination on the quantification assays. We compared the signal generated by $0.1 \mathrm{mg} / \mathrm{ml} \mathrm{CS}-\mathrm{A}$ and heparin in the presence of 0 to $0.2 \mathrm{mg} / \mathrm{ml}$ DNA. We measured two $10 \mu 1$ duplicates for each sample in the Alcian Blue assay and two $40 \mu 1$ duplicates for each sample in the carbazole assays. The results indicated that within this DNA concentration range, neither the Alcian Blue assay (Fig. 8A) nor the carbazole assay (Fig. 8B) was affected by DNA contamination.

\section{The Alcian Blue Assay is Sulfation Dependent}

Next we examined the impact of sulfation on the GAG assays. We used for this purpose three heparin species, each with a different degree of sulfation. Heparin is the most sulfated species, NDSNAc-heparin provides an intermediate degree of sulfation, and CDSNAc-heparin is the least sulfated species. The HPLC assay and the carbazole assays could detect all three different heparin species (Fig. 8D), and concentrations were the same when measured by the carbazole (Fig. 8D) and HPLC assays (HPLC data not shown). In contrast, the Alcian Blue assay did not detect CDSNAcheparin, the unsulfated species (Fig. 8C). This last result was consistent with a previous report which indicate that the Alcian Blue assay cannot detect HA, another unsulfated GAG [23]. The Alcian Blue assay did yield similar concentration response curves for sulfated GAGs ranging from 1 to 2.7 sulfates per disaccharide (Fig. 4A). Thus, the Alcian Blue assay can be used to quantify mixtures of sulfated GAGs.

Table 2. Quantification by HPLC and Carbazole Methods

\begin{tabular}{|c|c|c|c|c|c|c|c|}
\hline & $\begin{array}{c}\text { GlcN } \\
\text { (pmol) }\end{array}$ & $\begin{array}{c}\text { GalN } \\
(\mathrm{pmol})\end{array}$ & $\begin{array}{c}\text { GlcN+GalN } \\
\quad(\mathbf{p m o l})\end{array}$ & $\begin{array}{c}\text { GlcA } \\
(\mathrm{pmol})\end{array}$ & GlcN+GalN:GlcA ratio & $\begin{array}{c}\text { GAG, HPLC } \\
(\mathrm{mg} / \mathrm{ml})\end{array}$ & $\begin{array}{c}\text { GAG, Carbazole } \\
(\mathrm{mg} / \mathrm{ml})\end{array}$ \\
\hline CS-A & 201 & 1250 & 1452 & 2514 & 0.58 & 0.74 & 1.27 \\
\hline CS-C & 148 & 1208 & 1356 & 1375 & 0.99 & 0.69 & 0.70 \\
\hline CS-D & 0 & 1564 & 1564 & 1380 & 1.13 & 0.89 & 0.78 \\
\hline Heparin & 954 & 0 & 954 & 1278 & 0.75 & 0.61 & 0.82 \\
\hline
\end{tabular}



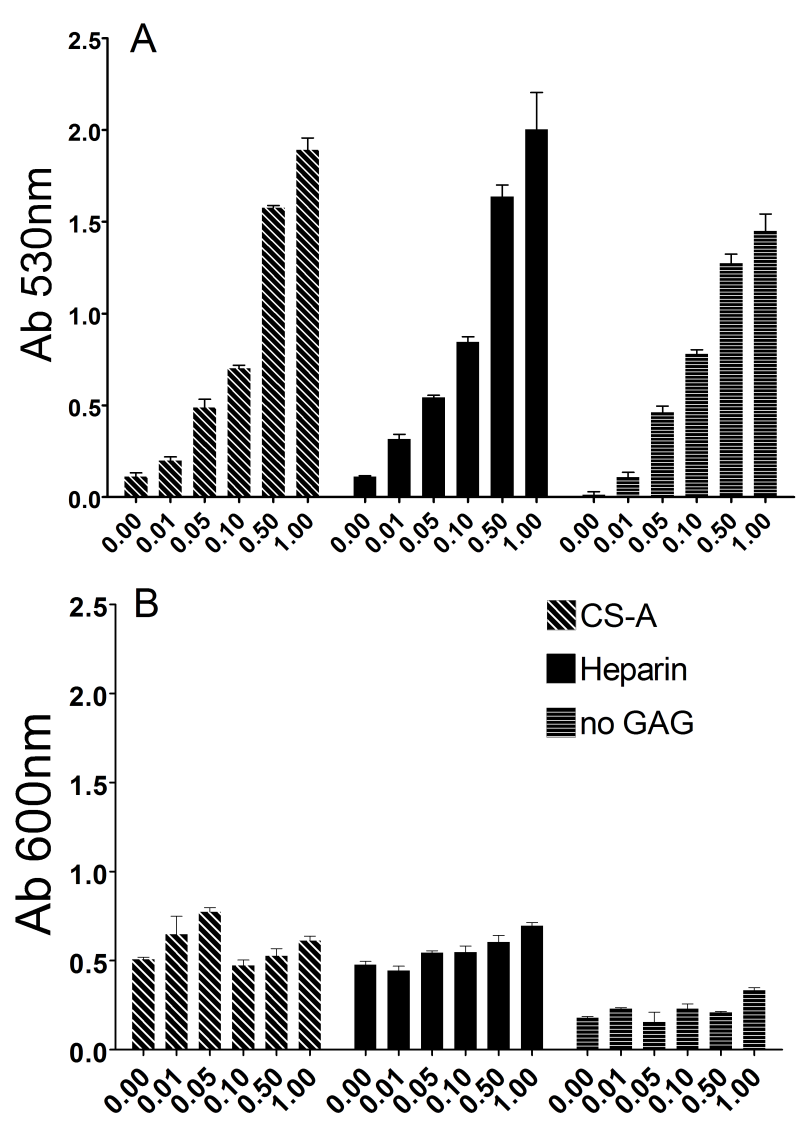

$[\mathrm{NaCl}](\mathrm{M})$ in $0.1 \mathrm{mg} / \mathrm{mL}$ GAG Stock Solution

Fig. (6). CS-A $(0.1 \mathrm{mg} / \mathrm{ml})$ or Heparin $(0.1 \mathrm{mg} / \mathrm{ml})$ was dissolved in $0,0.01,0.05,0.10,0.5$, and $1 \mathrm{M} \mathrm{NaCl}$ solutions. Control solutions contained $0.01,0.05,0.10,0.5$, and $1 \mathrm{M} \mathrm{NaCl}$ only. (A) $40 \mu 1$ duplicates of each solution were assayed by the carbazole method; (B) $10 \mu 1$ duplicates were assayed by the Alcian Blue method.

\section{DISCUSSION}

The HPLC assay is the most sensitive GAG quantification method available. It can be used to quantify the major classes of GAGs and can determine whether glucosamine GAGs, such as HS, KS, and HA, are contaminated with galactosamine GAGs, such as CS and DS or vice versa. However, the assay is time consuming and requires an HPLC with a fluorescence detector and Pico-tag hydrolysis station, instruments that are not available in most laboratories. Other methods have been developed for general carbohydrate analysis [26] and in some cases they can substitute for the HPLC method if the GAGs are completely hydrolyzed into non-sulfated uronic acid and glycosamine. This procedure also requires a Pico-tag hydrolysis station because GAGs are more difficult to hydrolyze than $\mathrm{N}$ - or O-linked oligosaccharides.

GAGs are relatively heterogeneous polysaccharides whose biological activities are usually determined by specific structural subsets. Thus the capacity to distinguish among GAG structural classes and detect possible GAGcross contamination can be essential in the elucidation of
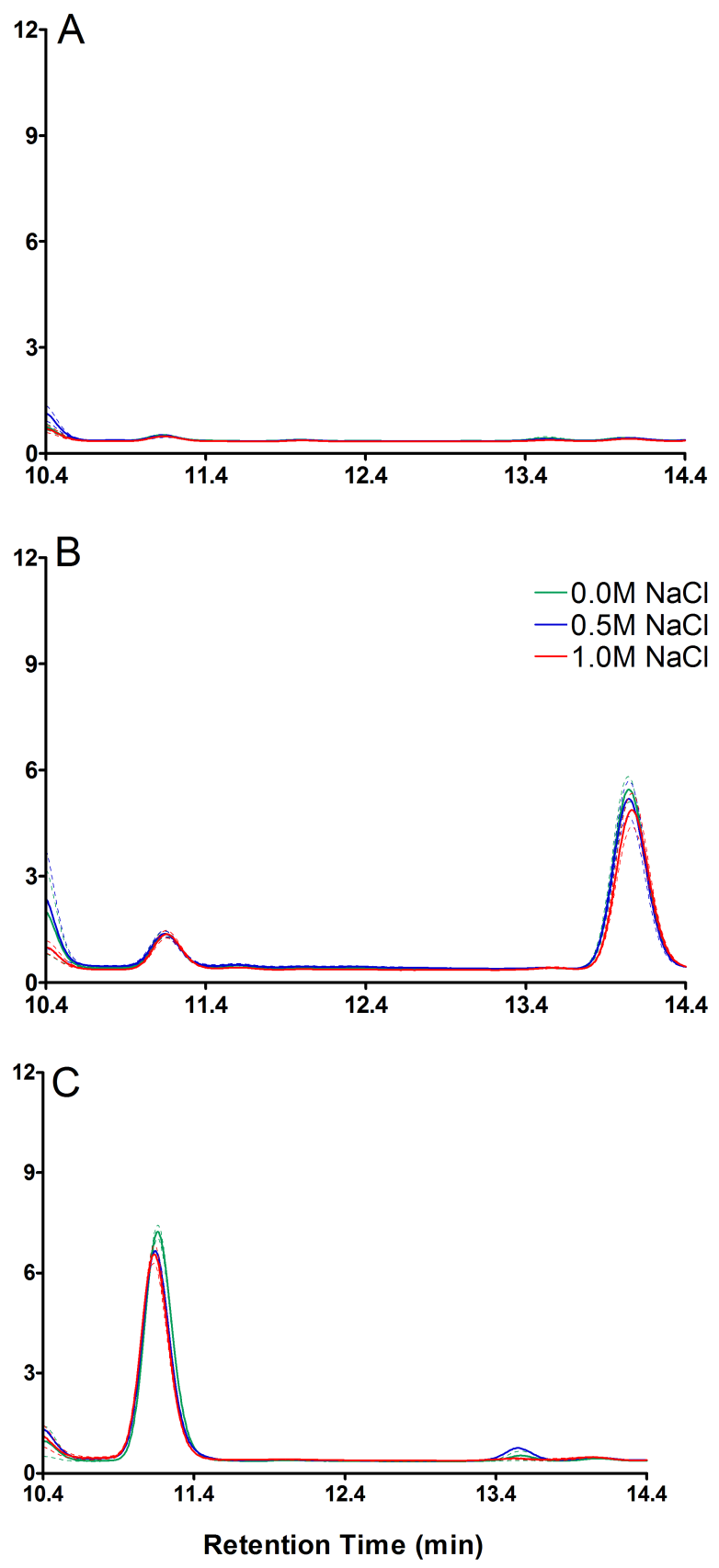

Fig. (7). CS-A $(0.01 \mathrm{mg} / \mathrm{ml})$ or heparin $(0.01 \mathrm{mg} / \mathrm{ml})$ was dissolved in $0,0.5$, and $1 \mathrm{M} \mathrm{NaCl}$ and $1 \mu 1$ duplicates were independently hydrolyzed and analyzed by the HPLC method.

GAG-ligand interactions. Not all GAG classes can be reliably detected by enzymatic degradation, for example it is well established that mammalian and bacterial hyaluronidase [27] and chondroitinase [28] digest both CS and HA. Similarly, nitrous acid treatment can be used to remove heparin, but it can also generate nitrous acid resistant HS fragments that can co-purify with other GAGs. Thus, the HPLC method remains the assay of the choice to distinguish among the major GAG structural groups.

Both the carbazole and the Alcian Blue assays [23] can be performed in most laboratory settings, although neither 

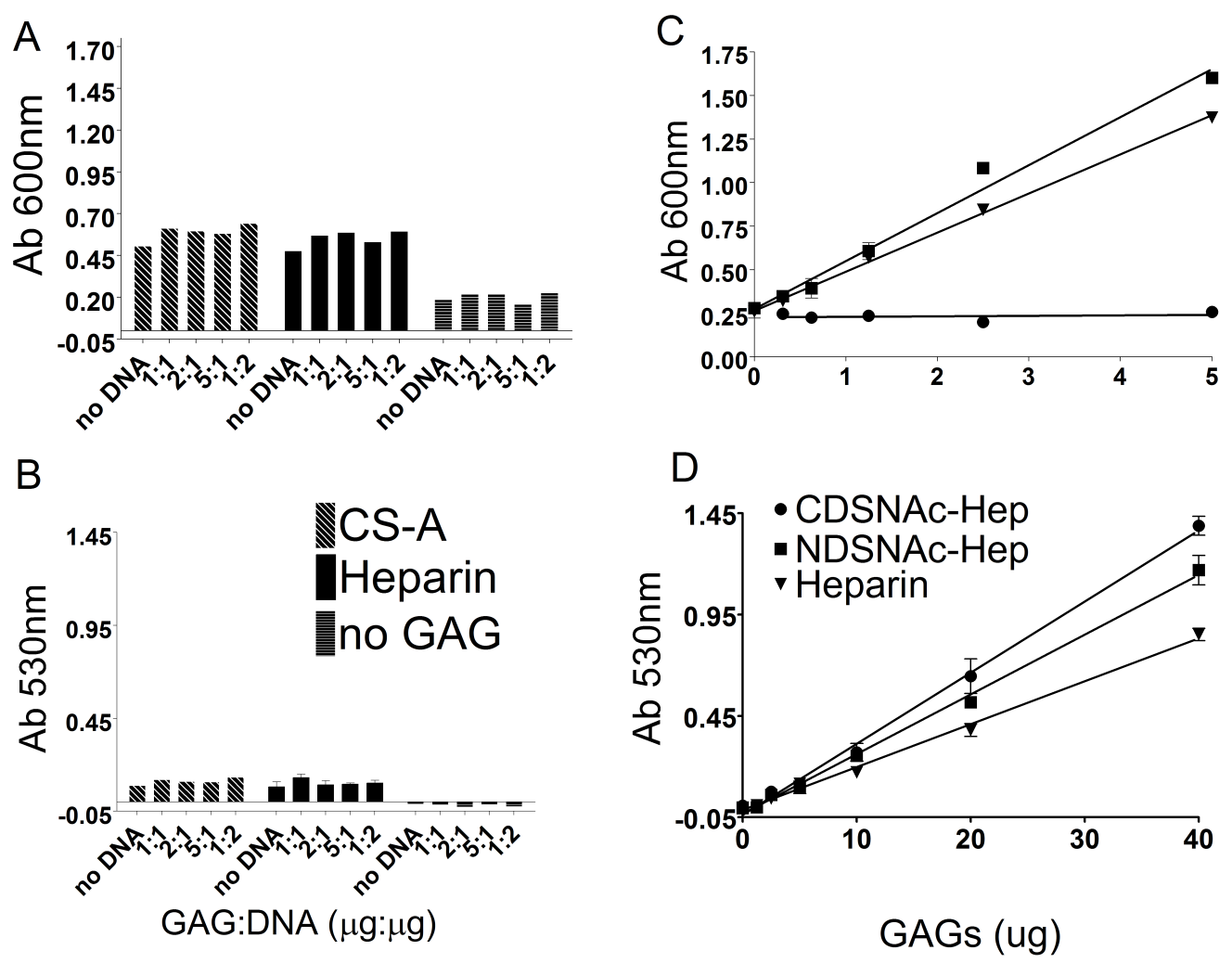

Fig. (8). CS-A $(0.1 \mathrm{mg} / \mathrm{ml})$ or heparin $(0.1 \mathrm{mg} / \mathrm{ml})$ was dissolved in solutions containing $0,0.02,0.05,0.1$, and $0.2 \mathrm{mg} / \mathrm{ml}$ DNA. Two $10 \mu 1$ duplicates of each solution were assayed by the Alcian Blue method (A) and two $40 \mu 1$ duplicates were assayed by the carbazole method (B). Heparin, NDSNAc-heparin, and CDSNAc-heparin were quantified by the Alcian Blue (C) and carbazole (D) assays. The data shown is representative of three independent experiments.

can distinguish between glucosamine- and galactosaminecontaining GAGs. The carbazole assay is based on the quantitative concentration-dependent color reaction between carbazole and uronic and can be as reliable as the HPLC assay for quantifying uronic acid containing GAGs [21,22]. However, the carbazole assay cannot detect $\mathrm{KS}$, which lacks uronic acid residues. We also observed that $\mathrm{NaCl}$ contamination generated false positive signals. Thus, it is essential that GAG samples must be salt-free. We observed previously that the carbazole assay overestimates concentration for GAGs that had undergone size exclusion chromatography in a salt containing buffer (Zhang L., unpublished data). The salt effect described in this report accounts for that observation. Since gel filtration is a common way of obtaining biologically active GAG oligosaccharide fragments, removing the salts before performing the carbazole assay must be included for achieving an accurate GAG quantification. Interestingly, Bitter and Muir also reported that the carbazole assay is sensitive to glucose, and that certain types of GAG signals are sensitive to chloride ions [21]. We conclude that the validity of the carbazole assay is largely dependent on GAG purity.

The sensitivity of our carbazole assay was 5-fold higher than that of the conventional assay $[21,22]$ due to a 5 -fold reduction in the reaction volume. It can detect as little as $1.25 \mathrm{nmol}(1.25 \mathrm{nmol} \times 194=243 \mathrm{ng})$ GlcA (Fig. 4) which produces a signal of $0.040 \pm 0.002$ absorbance units above the background. In contrast, the conventional carbazole assay consumes $\sim 2-5 \mu \mathrm{g}$ of GAGs. Cesaretti et al. also miniaturized the carbazole assay and adopted the same reaction vol- ume that we used, but in a 96-well format [29]. Surprisingly, the sensitivity of their assay is identical to the conventional tube assay, i.e., $1 \mu \mathrm{g}$ for GlcA and $3 \mu \mathrm{g}$ for GAGs. While they theoretically should have observed the same enhanced sensitivities that we did, it appears likely that the stringent temperature control required for thorough GAG hydrolysis and carbazole reactions cannot be achieved in a $100^{\circ} \mathrm{C}$ oven with an open lid 96-well format [29]. Indeed, when we adapted our reactions to their 96-well platform we observed a dramatic loss in GAG sensitivity (data not shown).

The Alcian Blue assay is the fastest way to quantify GAGs. Alcian Blue is a tetravalent cationic dye with a hydrophobic core that contains copper, which gives it its Blue color. Alcian Blue interacts with sulfated GAGs with high specificity at a pH low enough to neutralize all carboxyl and phosphoric acid groups and at an ionic strength great enough to eliminate ionic interactions other than those between Alcian Blue and sulfated GAGs. Based on the specific interactions between sulfated GAGs and Alcian Blue, the GAG quantification assay, in addition to other applications, has been developed [23].

The Alcian Blue protocol we used is 4-fold more sensitive than the miniaturized carbazole assay (Fig. 3) and the dot blot/reflection Alcian Blue assay [30] is even more sensitive. However, there are multiple issues associated with the Alcian Blue assay. First, Alcian Blue is not a well-defined compound; the solubility and GAG-binding characteristics of Alcian Blue not only differ among different manufacturers but also vary between batches from the same manufacturer. 
Thus, preliminary experiments are required to characterize the specific Alcian Blue dye before using it for quantification. Second, Alcian Blue-GAG interactions are sulfation and structure dependent; Alcian Blue does not bind nonsulfated GAGs [31]. Third, since this assay only detects sulfated polysaccharides, there is no monosaccharide or oligosaccharide "standard" GAG that can be used in the Alcian Blue assay to generate concentration response curves for GAG quantification. Therefore, the Alcian Blue assay is a "relative" GAG quantification method by nature.

In summary, the HPLC method should be the primary choice for quantification of both commercially available GAGs and GAGs purified from biological sources because it is extremely sensitive and it reliably distinguishes glucosamine- and galactosamine-containing GAGs. The carbazole assay is as reliable as the HPLC assay for the quantification of uronic acid containing GAGs, but the GAGs must be free of salts and other sugars such as glucose. The Alcian Blue assay is a fast and easy way to quantify GAGs but the values obtained by this assay should be interpreted with care because of its dependence on sulfation.

Multiple proteoglycans, such as syndecan, endocan, and glypican, and GAG structure editing enzymes, such as heparanase, sulfatases, and hyaluronidases, have been alleged to be cancer diagnosis and prognosis markers, or treatment targets [32-39]. The biological functions of proteoglycans are largely due their GAG chains. Thus, simple, sensitive, and robust GAG quantification methods are needed for the development of biomarkers. We suggest that an automated HPLC assay would be generally useful for the routine measurement of a panel of GAG-based biomarkers while the carbazole assay and the Alcian Blue assays could prove valuable for more specific purposes.

\section{MATERIALS \& METHODS}

\section{GAG Sources and Storage}

Heparin from porcine intestinal mucosa (H4784), CS-A from bovine trachea (C8529), CS-B from porcine intestinal mucosa (C3788), and CS-C from shark cartilage (C4384) were purchased from Sigma. CS-D rom Shark cartilage (Cat. \# 230689) was purchased from Calbiochem. Two $1 \mu \mathrm{g}$ samples of squid cartilage CS-E were purchased, one from Calbiochem (cat. \# 230690) and one from Seikagaku (Cat. \# 400678). A chemically modified heparin kit with completely desulfated, re-N-acetylated (CDSNAc) and N-sulfated, re-Nacetylated (NDSNAc) heparin was purchased from Seikagaku (Cat. \# 400645). HPLC analysis revealed that the NDSNAc-heparin had $\sim 3.8 \%$ GalN-containing GAG contaminations whereas no GalN was observed in the CDSNAcheparin. These results were in agreement with our early observations that GAG-cross contamination was batchdependent (Figs. 1 and 2).

We observed that GAG concentration gradually decreased when GAGs were stored at $4^{\circ} \mathrm{C}$ at a concentration of $1 \mathrm{mg} / \mathrm{ml}$. We made $10 \mathrm{mg} / \mathrm{ml}$ GAG aliquots in siliconized tubes and stored them at $-20^{\circ} \mathrm{C}$. For quantification purposes, we made fresh $1 \mathrm{mg} / \mathrm{ml}$ of GAG working solutions from the $10 \mathrm{mg} / \mathrm{ml}$ stock solution. For the carbazole and Alcian Blue assays, we made $200 \mu 1$ master serial dilution solutions in water containing $1 \mathrm{mg} / \mathrm{ml}, 0.5 \mathrm{mg} / \mathrm{ml}, 0.25 \mathrm{mg} / \mathrm{ml}, 0.125$ $\mathrm{mg} / \mathrm{ml}, 0.0625 \mathrm{mg} / \mathrm{ml}$, or $0.03125 \mathrm{mg} / \mathrm{ml}$ GAGs. Two $10 \mu 1$ duplicates of each GAG dilution were used for the Alcian Blue assay $(10 \mu \mathrm{g}, 5 \mu \mathrm{g}, 2.5 \mu \mathrm{g}, 1.25 \mu \mathrm{g}, 0.625 \mu \mathrm{g}$, and 0.3125 $\mu \mathrm{g}$, respectively) and two $40 \mu \mathrm{l}$ duplicates were used for the carbazole assay $(40 \mu \mathrm{g}, 20 \mu \mathrm{g}, 10 \mu \mathrm{g}, 5 \mu \mathrm{g}, 2.5 \mu \mathrm{g}$, and $1.25 \mu \mathrm{g}$, respectively).

\section{The HPLC Method}

GAGs were subjected to acid hydrolysis, sodiumborohydride reduction, precolumn derivatization with o-phthaldialdehyde (OPA) and 3-mercaptopropionic acid (3MPA), and reversed phase HPLC separation with fluorescence detection of the isoindole derivatives. GAG aliquots containing $360 \mathrm{pmol}$ of norleucine as an internal standard were dried in pyrolized glass vials (Agilent, Palo Alto, CA, part 51818872 ) before hydrolysis with $\mathrm{HCl}$ vapor in $\mathrm{N}_{2}$ gas at $100{ }^{\circ} \mathrm{C}$ for 3 hours. The samples were rehydrated in $45 \mu 1$ of $0.56 \%$ $\mathrm{NaBH}_{4}$ to reduce the glucosamine and galactosamine liberated by acid hydrolysis into glucosaminitol and galactosaminitol, respectively. After an overnight incubation at room temperature, the reaction was terminated by adding $5 \mu 1$ of $2 \mathrm{~N}$ acetic acid to each vial. The sample was dried by speedvac and dissolved in $5 \mu$ water for precolumn derivatization with $35 \mu \mathrm{l}$ of $7.5 \mathrm{mM}$ OPA, $375 \mathrm{mM} 3 \mathrm{MPA}$, in $0.4 \mathrm{~N}$ borate adjusted to $\mathrm{pH} 9.3$ with $\mathrm{NaOH}$. Half of this reaction mixture was injected onto a 4.6 x 250mm C-12 column, a Synergi $4 \mu$ MAX-RP $80 \AA$ (Phenomenex, Torrance, CA, part 00G-4337$\mathrm{E} 0$ ), and heated to $35^{\circ} \mathrm{C}$. The column was equilibrated with Buffer A, consisting of $0.05 \mathrm{M}$ (monobasic and dibasic) sodium phosphate, $\mathrm{pH} 7.2$ in $25 \%$ methanol, at a flow rate of $0.8 \mathrm{ml} / \mathrm{min}$. Buffer B consisted of methanol, water, and tetrahydrofuran at 70:30:3 volume ratios. After injection, Buffer $\mathrm{B}$ was increased from 0 to $8 \%$ by a linear gradient between 0 and $3 \mathrm{~min}$, was maintained at $8 \%$ between 3 and $18 \mathrm{~min}$, at $55 \%$ between 18 and $30.5 \mathrm{~min}$, at $100 \%$ between 30.5 and $32.5 \mathrm{~min}$, and at $0 \%$ between 32.5 and $35 \mathrm{~min}$. A $5 \mathrm{~min}$ post-run interval at $0 \% \mathrm{~B}$ preceded the initiation of the next precolumn derivatization injection sequence. The fluorescent derivatives of glucosaminitol, galactosaminitol, and the amino acids contained in the GAG preparations were excited at $337 \mathrm{~nm}$ and detected at $454 \mathrm{~nm}$. A more detailed description of this assay can be found in the original publication [20].

\section{The Carbazole Method}

Carbazole-based quantification involves two steps: 1.) hydrolyzing GAGs into uronic acid and glycosamine with $0.025 \mathrm{M}$ sodium tetraborate (Sigma) in sulfuric acid; and 2.) coloring uronic acid with carbazole (Sigma). Both reactions require boiling for $10 \mathrm{~min}$. A glass tube containing $3.6 \mathrm{ml}$ of reactants with a marble on top is usually used for the carbazole assay. The marble prevents splashing of sulfuric acid during boiling and the potential hazard caused by the tube's cap popping. The assay sensitivity is $1 \mu \mathrm{g}$ for GlcA and $3 \mu \mathrm{g}$ for GAGs. For GAG quantification, at least two $\sim 5 \mu \mathrm{g}$ duplicate samples of unknown the GAG are required. We reasoned that if we scaled down the reaction volume, the same amount of GAGs would generate more concentrated colors, and thus enhance the assay sensitivity of this assay.

We used a PCR instrument set at $100^{\circ} \mathrm{C}$ for the hydrolysis and carbazole reactions. The reagents used in the carba- 
zole assay were $0.025 \mathrm{M}$ sodium tetraborate $10 \mathrm{H}_{2} \mathrm{O}$ in sulfuric acid $\left(\mathrm{H}_{2} \mathrm{SO}_{4}+\right.$ Borex $)$ and $0.125 \%$ carbazole in absolute ethanol (W/V). We added $200 \mu \mathrm{l}$ of the $\mathrm{H}_{2} \mathrm{SO}_{4}+$ Borex solution to $40 \mu \mathrm{l}$ of GAG solution or water (blank control). The tubes were capped and vortexed briefly. The tubes were then put into a PCR instrument with the lid securely locked. The PCR instrument was programmed to run for $15 \mathrm{~min}$ at $100^{\circ} \mathrm{C}$ and then cool down to $4^{\circ} \mathrm{C}$. To each tube, $8 \mu 1$ of carbazole solution was added followed by a brief vortex. The tubes were returned to the PCR instrument for the carbazole reaction, which was run at $15 \mathrm{~min}$ at $100^{\circ} \mathrm{C}$ and then cooled to $4^{\circ} \mathrm{C}$. The samples were vortexed and $200 \mu 1$ of each sample was transferred to a 96 well plate. The absorbance at $530 \mathrm{~nm}$ was measured by a Spectra MAX M2 plate-reading spectrophotometer (Molecular Devices, Sunnyvale, CA). GlcA was used as an external standard, and the standard curve was derived by plotting absorbance against the concentration of GlcA or standard GAG.

\section{The Alcian Blue Method}

We used a simpler Alcian Blue assay [24] than the assay described by Karlsson and Bjornsson [23]. In brief, the Alcian Blue dye stock solution consisted of a 1/100 dilution of the original dye solution (1g Fluka Alcian Blue 8GS with $100 \mathrm{ml}$ of $18 \mathrm{mM} \mathrm{H}_{2} \mathrm{SO}_{4}$ ). The $1 / 100$ dilution was brought to an absorbance of $\sim 1.4$ at $600 \mathrm{~nm}$ by adding additional dye (if needed). This was followed by centrifugation at $10,000 \mathrm{rpm}$ for $30 \mathrm{~min}$ to remove insoluble dye particles. The working dye solution containing $0.25 \%$ Triton X-100, $0.018 \mathrm{M} \mathrm{H}_{2} \mathrm{SO}_{4}$ and $10 \%$ dye stock was made and filtered $(0.2 \mu \mathrm{m}$ filter $)$. $10 \mu \mathrm{l}$ of a solution containing $0.027 \mathrm{M} \mathrm{H}_{2} \mathrm{SO}_{4}, 0.375 \%$ Triton $\mathrm{X}-100$, and $4 \mathrm{M}$ guanidine $\mathrm{HCl}$ was added to each $10 \mu \mathrm{l}$ aliquot of a GAG standard series $(0,0.3175 \mu \mathrm{g}, 0.625 \mu \mathrm{g}$, $1.25 \mu \mathrm{g}, 2.5 \mu \mathrm{g}, 5 \mu \mathrm{g}$, and $10 \mu \mathrm{g}$ in $10 \mu \mathrm{l}$ of water) along with $100 \mu 1$ of working dye solution. After microcentrifugation for $10 \mathrm{~min}$ at $16000 \mathrm{~g}$ at $4^{\circ} \mathrm{C}$, the supernatant was aspirated and the pellet was dissolved in $500 \mu \mathrm{l}$ of $8 \mathrm{M}$ guanidine $\mathrm{HCl}$ by rigorous vortexing.

The samples were centrifuged for $3 \mathrm{~min}$ at $16000 \mathrm{~g}$. $300 \mu \mathrm{l}$ of supernatant was transferred to a $96-w e l l$ plate. The absorbance at $600 \mathrm{~nm}$ was measured using a Spectra MAX M2 plate-reading spectrophotometer.

\section{Determining Concentration Response Curves}

The concentration response curves for both the carbazole and the Alcian Blue assays were plotted using the GraphPad Prism software. The software automatically formulated the equations, in which $\mathrm{A}$ is equal to measured absorbance and $\mathrm{c}$ is equal to the amount of GAGs expressed in $\mu \mathrm{g}$. The base line absorbance readings and equations changed from day to day due to changes in reagents, sample preparations, or other unknown factors, but the $r^{2}$ values of both assays were always $\geq 0.98$, indicating their reliability.

\section{Comparison of GAG Quantity as Determined by the HPLC And Carbazole Assays}

We calculated the A value for $1 \mu \mathrm{g}$ of each GAG by using the corresponding carbazole curve fitting equation shown in Fig. (4B). We then used the A values in the GlcA equation, $c$ $=26.78 A+0.4524$, to calculate equivalent GlcA quantities based on activity. The HPLC method includes an internal standard, norleucine (NorL), and a series of external GlcN-
$\mathrm{OH}$ and GalN-OH standards. Based on the calibration curves of the GlcN-OH and GalN-OH, the nmols of GlcN and GalN equivalent to the fluorescence units generated by $1 \mu \mathrm{g}$ (dry weight) of GAGs were calculated (Table 2). The $1 \mu \mathrm{g}$ GAG equivalent pmols of GlcA and the ratio of GlcN+GalN (as quantified by HPLC) to GlcA for each GAG was summarized in Table 2. The GlcA values plotted in Fig. (4B) were 2524,906 , and $861 \mathrm{pmol}$ for 1,10 , and $40 \mu \mathrm{g}$ of CS-A respectively. $2524 \mathrm{pmol}$ value was used to calculate the absorbance (A) value.

\section{Molecular Weights Of GAG Dissacharides}

An analysis of GAG disaccharides by capillary HPLC/MS (not shown) indicated that CS-A, CS-B, and CS$\mathrm{C}$ had $\sim 1$ sulfate per repeating disaccharide; CS-D and CS-E had $\sim 1.6$ sulfates per disaccharides; and heparin had 2.7 sulfates per disaccharide. Because all of the GAGs are in the sodium form, the $\mathrm{mg} / \mathrm{ml} \mathrm{GAG} \mathrm{concentrations} \mathrm{were} \mathrm{calcu-}$ lated based on F.W. 505 (379 for repeating disaccharides + 80 for sulfate $+2 \times 23$ for sodium) per disaccharide for CSA, CS-B, and CS-C; $566.8(379+1.6 \times 80+2.6 \times 23)$ per disaccharide for CS-D and CS-E, and $638(337+2.7 \times 80+$ $3.7 \times 23$ ) per disaccharide for heparin.

\section{Low pH Nitrous Treatment of HS-Containing CS-B}

A standard low $\mathrm{pH}$ nitrous treatment procedure was used to remove HS from the contaminated CS-B [25]. In brief, CS-B was treated with $\mathrm{HNO}_{2}$ at $\mathrm{pH} 1.5$ for 30 minutes on ice. The treated CS-B sample was dialyzed against water in $5 \mathrm{~K}$ cut-off dialysis tubing for two days and then dried and re-dissolved in water.

\section{Preparation of Oligosaccharides from CS-B}

The CS-B oligosaccharides were prepared by a previously published procedure [40]. In brief, both purified and contaminated samples of CS-B were partially deacetylated with hydrazine/hydrazine sulfate and then reacted with high $\mathrm{pH}$ nitrous acid, which quantitatively cleaves the glycosidic linkages following $\mathrm{N}$-unsubstituted $\mathrm{D}$-galactosamine residues ( $\mathrm{N}$-acetyl-D-glycosamine residues are resistant to deaminative cleavage at high $\mathrm{pH}$ ). This procedure yields oligosaccharide mixtures.

The mixtures were then separated into different oligosaccharides by gel filtration on a Bio-Gel P-10 column. Aliquots of purified oligosaccharides were then quantified by the HPLC method.

\section{ACKNOWLEDGEMENTS}

We thank Dr. Douglas D. Tollefsen for his insightful suggestions and critiques of the manuscript. This work is supported in part by National Institutes of Health Grant: R01GM069968.

\section{REFERENCES}

[1] Toleffsen DM, Zhang L. In: Colman RW, Ed. Hemostasis and Thrombosis. Lippincott Williams \& Wilkins 2006; 271-83.

[2] Robertson MS. Heparin: the cheap alternative for immunomodulation in sepsis? Crit Care Resusc 2006; 8: 235-38.

[3] Girardi G. Heparin treatment in pregnancy loss: Potential therapeutic benefits beyond antigcoagulation. J Reprod Immunol 2005; 66: 45-51.

[4] Chancellor MB, Yoshimura N. Treatment of interstitial cystitis. Urology 2004; 63: 85-92. 
[5] Falanga A. The effect of anticoagulant drugs on cancer. J Thromb Haemost 2004; 2: 1263-5.

[6] Gao Y, Li N, Fei R, Chen Z, Zheng S, Zeng X. P-Selectin-mediated acute inflammation can be blocked by chemically modified heparin, RO-heparin. Mol Cells 2005; 19: 350-55.

[7] Varki NM, Varki A. Heparin inhibition of selectin-mediated interactions during the hematogenous phase of carcinoma metastasis: rationale for clinical studies in humans. Semin Thromb Hemost 2002; 28: 53-66.

[8] Wang L, Brown JR, Varki A, Esko JD. Heparin's anti-inflammatory effects require glucosamine 6-O-sulfation and are mediated by blockade of L- and P-selectins. J Clin Invest 2002; 110: 127-36.

[9] Volpi N. Therapeutic applications of glycosaminoglycans. Curr Med Chem 2006; 13: 1799-810.

[10] Conrad HE. Heparin-Binding Proteins. San Diego: Academic Press 1998.

[11] Bishop JR, Schuksz M, Esko JD. Heparan sulphate proteoglycans fine-tune mammalian physiology. Nature 2007; 446: 1030-7.

[12] Godden JL, Edward M, MacKie RM. Melanoma cell-derived facter stimulation of fibroblast glycosaminoglycan synthesis - the role of platelet-derived growth facter. Eur J Cancer 1999; 35: 473-80.

[13] Prante C, Milting H, Kassner A, et al. Transforming growth factor beta 1-regulated xylosyltransferase I activity in human cardiac fibroblasts and its impact for myocardial remodeling. J Biol Chem 2007; 282: 26441-9.

[14] Guerrini M, Naggi A, Guglieri S, Santarsiero R, Torri G. Complex glycosaminoglycans: profiling substitution patterns by twodimensional nuclear magnetic resonance spectroscopy. Anal Biochem 2005; 337: 35-47.

[15] Saad OM, Leary JA. Compositional analysis and quantification of heparin and heparan sulfate by electrospray ionization ion trap mass spectrometry. Anal Chem 2003; 75: 2985-95.

[16] Kuberan B, Lech M, Zhang L, Wu ZL, Beeler DL, Rosenberg RD. Analysis of heparan sulfate oligosaccharides with ion pair-reverse phase capillary high performance liquid chromatographymicroelectrospray ionization time-of-flight mass spectrometry. J Am Chem Soc 2002; 124: 8707-18.

[17] Toyoda H, Kinoshita-Toyoda A, Fox B, Selleck SB. Structural analysis of glycosaminoglycans in animls bearing mutations in sugarless, and tout-velu. Drosophila homologues of vertebrate genes encoding glycosaminoglycan biosynthetic enzymes. J Biol Chem 2000; 275: 21856-61.

[18] Volpi N, Maccari F. Electrophoretic approaches to the analysis of complex polysaccharides. J Chromatogr B 2006; 834: 1-13.

[19] Lee YC. High-performance anion-exchange chromotography for carbohydrate analysis. Anal Biochem 1990; 189: 151-62.

[20] Studelska DR, Giljum K, McDowell LM, Zhang L. Quantification of glycosaminoglycans by reversed-phase HPLC separation of fluorescent isoindole derivatives. Glycobiology 2006: 16; 65-72.

[21] Bitter T, Muir HM. A modified uronic acid carbazole reaction. Anal Biochem 1962; 4: 330-4.

[22] Plaetzer M, Ozegowski JH, Neubert RH. Quantification of hyaluronan in pharmaceutical formulations using high performance capillary electrophoresis and the modified uronic acid carbazole reaction. J Pharm Biomed Anal 1999; 21: 491-96.

[23] Karlsson M, Bjornsson S. Quantification of proteoglycans in biological fluids using Alcian blue. Methods Mol Biol 2001; 171: 15973.
[24] Zhang L, Lawrence R, Schwartz JJ, et al. The effect of precursor structures on the action of 3-OST-1 and the biosynthesis of anticoagulant heparan sulfate. J Biol Chem 2001; 276: 28806-13.

[25] Guo YC, Conrad HE. Analysis of oligosaccharides from heparin by reversed=phase ion-pairing high-perofrmance liquid chromatography. Anal Biochem 1988; 168: 54-62.

[26] Campo GM, Campo S, Ferlazzo AM, Vinci R, Calatroni A. Improved high-performance liquid chromatographic method to estimate aminosugars and its application to glycosaminoglycan determination in plasma and serum. J Chromatogr B Biomed Sci Appl 2001; 765: $151-60$.

[27] Sugahara K, Tanaka Y, Yamada S. Preparation of a series of sulfated tetrasaccharides from shark cartilage chondroitin sulfate D using testicular hyaluronidase and structure determination by 500 MHz ${ }^{1}$ H NMR spectroscopy. Glycoconj J 1996; 13: 609-19.

[28] Huang W, Lunin VV, Li Y, et al. Crystal Structure of Proteus vulgaris Chondroitin Sulfate ABC Lyase I at 1.9 A resolution. J Mol Biol 2003; 328: 623-34.

[29] Cesaretti M, Luppi E, Maccari F, Volpi N. A 96-well assay for uronic acid carbazole reaction. Carbohydr Polym 2003; 54: 59-61.

[30] Bjornsson S. Quantitation of proteoglycans as glycosaminoglycans in biological fluids using an Alcian blue dot blot analysis. Anal Biochem 1998; 256: 229-37.

[31] Bjornsson S. Simultaneous preparation and quantitation of proteoglycans by precipitation with alcian blue. Anal Biochem 1993; 210: 282-91.

[32] Anttonen A, Leppae S, Ruotsalainen T, Alfthan H, Mattson K, Joensuu H. Pretreatment serum syndecan-1 levels and outcome in small cell lung cancer patients treated with platinum-based chemotherapy. Lung Cancer 2003; 41: 171-7.

[33] Fuster MM, Esko JD. The sweet and sour of cancer: glycans as novel therapeutic targets. Nat Rev Cancer 2005; 5: 526-42.

[34] Grigoriu BD, Depontieu F, Scherpereel A, et al. Endocan expression and relationship with survival in human non-small cell lung cancer. Clin Cancer Res 2006; 12: 4575-82.

[35] Kim H, Xu GL, Borczuk AC, et al. The heparan sulfate proteoglycan GPC3 is a potential lung tumor suppressor. Am J Respir Cell Mol Biol 2003; 29: 694-701.

[36] Lokeshwar VB, Rubinowicz D, Schroeder GL, et al. Stromal and Epithelial expression of tumor markers Myaluronic acid and HYAL1 lyaluronidase in prostate cancer. J Biol Chem 2001; 276: 11922-32.

[37] Morimoto-Tomita M, Uchimura K, Bistrup A, et al. Sulf-2, a proangiogenic heparan sulfate endosulfatase, is upregulated in breast cancer. Neoplasia 2005; $7:$ 1001-10.

[38] Vlodavsky I, Friedmann Y. Molecular properties and involvement of heparanase in cancer metastasis and angiogenesis. J Clin Invest 2001; 108: 341-7.

[39] Yang Y, Macleod V, Miao HQ, et al. Heparanase enhances syndecan-1 shedding: a novel mechanism for stimulation of tumor growth and metastasis. J Biol Chem 2007; 282: 13326-33.

[40] Maimone MM, Tollefsen DM. Structure of dermatan sulfate hexasaccharide that binds to heparin cofactor II with high affinity. J Biol Chem 1990; 265: 18263-71. 